\title{
O comportamento materno e a depressão pós-parto no desenvolvimento prossocial em crianças de 36 meses de idade
}

\author{
Laura Cristina Stobäus ${ }^{1}$ \\ Beatriz Servilha Brocchi ${ }^{2}$ \\ Vera Silvia Raad Bussab ${ }^{3}$ \\ ${ }^{1}$ Pontifíicia Universidade Católica do Rio de Janeiro, RJ, Brasil \\ ${ }_{2}^{2}$ Pontificia Universidade Católica de Campinas, SP, Brasil \\ ${ }^{3}$ Universidade de São Paulo, SP, Brasil
}

\begin{abstract}
Resumo
Esta pesquisa teve como objetivo verificar a influência da depressão pós-parto no comportamento materno no desenvolvimento da linguagem e comportamento prossocial de crianças de 3 anos de idade. Participaram desta pesquisa 61 díades mães-crianças atendidas pelo Sistema Público de Saúde do Município de São Paulo, no qual 28 mães apresentaram indicativos de depressão em algum momento após o nascimento do filho, em uma das avaliações realizadas (puerpério, 8, 24 e aos 36 meses) no projeto longitudinal. Avaliou-se o aspecto pragmático do desenvolvimento de linguagem (por atos e meios comunicativos) e a interação mãe e criança na tarefa de recolher os brinquedos, em filmagens de vinte minutos de brincadeira livre. Os dados, analisados por meio de testes estatísticos paramétricos, apontam que os fatores da depressão que influenciam o comportamento materno na relação com seu filho são sutis, pois as crianças apresentaram um desempenho linguístico dentro dos padrões esperados para a idade, verbalizaram mais e usaram mais gestos para se comunicar, exibiram mais objetos às suas mães e comentaram mais. As mães sem depressão explicam mais a seus filhos as razões e os motivos e seus filhos fornecem menos ajuda às suas mães, demonstrados por meio de recusas e desafios.
\end{abstract}

Palavras-chave: Depressão materna; Comportamento prossocial; Desenvolvimento da pragmática.

\section{The maternal behavior and the post-partum depression in the development of language and prossocial behavior of 3-year-old children}

\begin{abstract}
This study aimed at verifying the influence of post-partum depression on the maternal behavior in the development of language and prossocial behavior of 3-year-old children. A total of 61 mother-child dyads being attended by the Public Health System in the Municipality of São Paulo took part in this study, where 28 mothers presented signs of depression at some point after childbirth in one of the assessments performed (puerperium, 8, 24 and 36 months) during the longitudinal project. During twenty minutes of free play between mother and child, pragmatic aspect of language development (through communicative actions and means) and the clean-up task were assessed. The data, analyzed through parametric statistical tests, showed that depression factors influencing maternal behavior in relation to the child are subtle, insofar as the children presented a linguistic performance within the standards expected for the age, verbalized more and used more gestures to communicate, exhibiting more objects to their mothers and made more comments. Mothers without depression explain the reasons and motives of things to their children and they, in turn, provide less help to their mothers, which is demonstrated through defiance and refusals.
\end{abstract}

Keywords: Maternal depression; Prossocial behavior; Pragmatic development.

\section{El comportamiento materno y la depresión posparto en el desarrollo del lenguaje y comportamiento prosocial de niños de 3 años de edad}

\section{Resumen}

Esta investigación tuvo por objetivo verificar la influencia de la depresión posparto en el comportamiento materno en el desarrollo del lenguaje y comportamiento prosocial de niños de 3 años de edad. En el presente estudio, 61 mujeres murieron atendidas por el Sistema Público de Salud del Municipio de São Paulo, en las cuales 28 madres presentaron indicativos de depresión en algún momento después del nacimiento del hijo en una de las evaluaciones realizadas (puerperio, 8, 24 y 36 Meses) en el diseño longitudinal. Se evaluó el aspecto pragmático del desarrollo del lenguaje (por actos y medios comunicativos) y la interacción madre y niño en la tarea de recoger los juguetes, en filmaciones de vinte minutos de juego libre. Los datos, analizados a través de pruebas estadísticas paramétricas, apuntan que los factores de la depresión que influencian el comportamiento materno en la relación con su hijo son sutiles, pues los niños presentaron un desempeño lingüístico dentro de los estándares esperados para la edad, verbalizaron más y utilizaron más gestos para comunicarse, exhibieron más objetos a sus madres y comentaron más. Las madres sin depresión explican más a sus hijos las razones y los motivos y sus hijos proporcionan menos ayuda a sus madres, demostradas por medio de rechazos y desafíos.

Palabras clave: Depresión materna; Comportamiento pro social; Desarrollo de la pragmática. 


\section{Introdução}

A depressão pós-parto caracteriza-se por um episódio depressivo ou uma soma de episódios que se iniciam geralmente entre a quarta e oitava semanas após o parto, alcançando sua intensidade máxima nos seis primeiros meses (Klaus \& Klaus, 2001; Schwengber \& Piccinini 2003). Possui etiologia multifatorial, sem formar um quadro homogêneo (Hatzinikolaou \& Murray, 2010; Schmidt, Piccoloto \& Müller, 2005). A mulher com depressão pós-parto pode apresentar alguns dos seguintes sintomas (podendo ter início na gestação): 1. humor deprimido e tristeza; 2. perda de interesse nas atividades diárias; 3 . alterações do sono (insônia ou hipersônia); 4. perda ou aumento de apetite e de peso; 5. fadiga e sensação de diminuição da energia; 6. agitação ou lentidão psicomotora; 7. sentimentos de culpa e de desmerecimento; 8. comportamento suicida (que podem variar entre pensamentos, planos e tentativas); 9. dificuldade para pensar, concentrarse ou tomar decisões. O diagnóstico é feito quando, no mínimo, cinco desses sintomas são identificados, sendo que um deles deve ser tristeza ou diminuição de interesse (ou prazer) em quase todas as atividades, que os sintomas permaneçam por, no mínimo, duas semanas e que estes causem sofrimento ou prejuízo no funcionamento social, ocupacional ou em outras áreas da vida do indivíduo (DSM V - APA, 2014).

Estudos em diferentes ambientes socioculturais têm mostrado uma incidência de depressão nos primeiros seis meses pós-parto (DPP) em torno de 10 a $20 \%$. A prevalência de depressão materna em países de renda baixa e média é estimada de 15 a $28 \%$ na África e Ásia, 28 a 57\% no Paquistão e 35 a 50\% na América Latina (Silva, 2013). A prevalência relativamente alta de depressão materna nesses países pode estar relacionada a vários fatores de risco como: conflitos, desastres, violência, imigração e alta prevalência de AIDS (Silva, 2013; Wachs, Black \& Engle, 2009). Brum e Schermann (2006) investigaram a prevalência da depressão pós-parto materna no Rio Grande do Sul (Brasil) e encontraram o índice de 19,1\%. Trabalhos utilizando a Escala de Depressão Pós-Parto de Edimburgo relatam prevalências de $12 \%$ a $37,1 \%$ de na população brasileira (Da Silva et al., 1998; Skafuzca, 2000; Zaconeta, Motta \& França, 2004; Cruz, Simões, Faisal-Cury, 2005; Fonseca, Silva, Otta, 2010).

A depressão pós-parto pode ser prejudicial ao vínculo mãe-bebê e é considerada um fator de risco, pois compromete a disponibilidade cognitiva e emocional da mãe, caracterizado como comportamentos parentais disfuncionais (Goodman \& Garber, 2017) no qual a mãe não seja adequadamente responsiva à criança, pela falta de contingência, de engajamento e de trocas ajustadas. Schwengber e Piccinini (2003) acrescentam que as mães que apresentam sintomas depressivos estão relacionadas à pobreza de interação, à ausência ou a pouca harmonização afetiva. Estas mães podem estar inseguras em suas capacidades maternas, gastam menos tempo olhando, tocando, falando com seus bebês, apresentam mais expressões negativas do que positivas, mostram menos responsividade, espontaneidade e menores índices de atividade (Kuczynski \& Kochanska, 1990; Lovejoy, Grackyk, O'Hare \& Neuman, 2000; Schwengber \& Piccinini, 2005). Dessa forma, o bebê pode experimentar uma desorganização comportamental, diminuindo o nível de respostas contingentes com relação à mãe.

Como um processo de adaptação à dificuldade de se relacionar com uma mãe deprimida, a criança pode apresentar padrões de comportamentos hostis ao interagir com ela, torna-se mais agitada ou arredia (Field et al., 2010; Tronick \& Gianino, 1986) e se relaciona com pares de um modo que não é bem adaptado (Goodman, Brogan, Lynch \& Fielding, 1993). Os bebês, segundo diversos autores, podem apresentar: 1) mais afeto negativo (expressões de tristeza, raiva ou tendência à irritabilidade); 2) menos responsivos nas relações interpessoais); 3) desenvolvimento motor mais precário; 4) em idade pré-escolar, dificuldades para se envolver e manter uma interação social, tendo déficits na regulação dos seus estados afetivos; 5) maiores dificuldades alimentares e de sono (Motta, Lucion \& Manfro, 2005; Schmidt et al., 2011; Lopes et al., 2010). A pesquisa de Radke-Yarrow, Cummings, Kuczynski e Chapman (1985) mostrou, por sua vez, que as crianças de mães deprimidas possuíam uma forma particular de lidar com suas mães, com preocupação excessiva com seus pais e em serem bons filhos.

A exposição da criança a estilos de interação não adequados pode representar diferentes tipos de risco para o desenvolvimento afetivo e cognitivo da criança, como: depressão, baixa estima, dificuldades escolares e de interação social, alterações linguísticas, entre outras (Seidl-de-Moura \& Ribas, 2004; Schwengber \& Piccinini, 2003). Com relação aos aspectos linguísticos, Motta et al. (2005), em sua revisão, relataram que as crianças filhas de mães depressivas apresentam linguagem expressiva menos rica que as crianças cujas mães não apresentam depressão. Quevedo et al. (2011) realizaram um estudo cujo objetivo foi analisar o efeito da duração da depressão materna no desenvolvimento da linguagem de crianças brasileiras de 12 meses de idade. Foram acompanhadas 296 díades, em dois encontros: um de 30 a 60 dias após o parto e o outro aos 12 meses. Foi observado que as crianças, cujas 
mães apresentavam sintomas de DPP, apresentavam risco maior de aquisição de linguagem aos 12 meses. $\mathrm{E}$, quando os sintomas depressivos persistiam, as crianças apresentavam escores menores na escala de linguagem, em comparação àquelas que eram expostas aos sintomas depressivos apenas em um momento específico. As autoras relatam que a exposição das crianças à depressão pode ser preditor para problemas de linguagem e seu efeito acumulativo de exposição pode trazer mais prejuízos, com relação à interação, acarretando em problemas de comportamento e vocabulário.

Brocchi e Bussab (2015) complementam esses achados em um estudo realizado com 80 díades mãescrianças atendidas pelo sistema público de saúde: dentre as mães, 30 apresentaram indicativos de DPP. O objetivo da pesquisa foi verificar a influência da depressão pós-parto e do contexto afetivo de criação no desenvolvimento da linguagem de crianças de 36 meses. Embora a diferença não tenha sido significativa, o grupo de crianças de mães sem sintomas depressivos obteve melhor desempenho, pois interagiu mais com sua mãe e, proporcionalmente verbalizou mais que o outro grupo, que, por sua vez, utilizou mais gestos e vocalizações.

Dessa forma, a depressão afeta a criança pela alteração dos modelos de interação diádica pois, como dito anteriormente, a criança não é considerada passiva, mas um participante ativo na formação de suas trajetórias. A exposição da criança a estilos de interação não adequados representa diferentes tipos de risco para o desenvolvimento afetivo e cognitivo da criança.

Com ênfase no entendimento dos fatores relevantes para o desenvolvimento infantil o presente projeto está inserido no Projeto Temático FAPESP (no 06/59192), conhecido como Projeto Ipê, "Depressão pós-parto como um fator de risco para o desenvolvimento do bebê: estudo interdisciplinar dos fatores envolvidos na gênese do quadro e em suas consequências". Este estudo objetivou verificar as influências da DPP nas práticas parentais e no desenvolvimento da linguagem em crianças de 3 anos. A análise destes efeitos foi feita dentro de um contexto mais amplo de variáveis relacionadas à composição familiar e ao apoio social, o que permitiu aprofundar a compreensão dos processos subjacentes.

\section{Método}

\section{Participantes}

Participaram desta pesquisa 61 díades mães-crianças da cidade de São Paulo, cujas crianças participantes tinham idade de trinta e seis meses. De acordo com a Escala de Depressão Pós-parto de Edinburgo, $28(45,9 \%)$ das mães apresentaram indicativos de depressão, no qual $13(21,3 \%)$ apresentaram apenas um episódio (no puerpério) e 15 (24,6\%) mais de um episódio em uma das avaliações realizadas (além do puerpério aos 8 meses, 24 ou 36 meses) no projeto longitudinal.

Foram incluídas no estudo todas as crianças de 36 meses que realizaram todas as avaliações do estudo longitudinal, cujas mães foram avaliadas através da Escala de Edinburgo em todas as etapas do projeto. As mães com DPP foram incluídas quando apresentaram um ou mais de um episódio em uma das avaliações realizadas durante a pesquisa. Foram excluídas as crianças que não apresentavam a faixa etária do estudo, que não realizaram a filmagem, crianças gemelares, com alterações auditivas, visuais, neurológicas, síndromes. Foram excluídas também as mães que não foram avaliadas em alguma das etapas do estudo.

\section{Instrumentos}

Foram utilizados na pesquisa os seguintes instrumentos:

\section{Aplicados à mãe}

Escala de Depressão Pós-parto de Edinburgo (EDPE), de Cox, Holden e Sagovsky (1987), validada no Brasil por Santos, Martins e Pasquali (1999) é utilizada para rastrear a prevalência de depressão pós-parto (escores $\geq 12$ ). A EDPE é um instrumento de auto-preenchimento composto por 10 enunciados (ex: Eu tenho sido capaz de rir e achar graça das coisas; Eu tenho me sentido esmagada pelas tarefas e acontecimentos do meu dia-a-dia; Eu tenho me sentido triste ou arrasada) cujas opções são pontuadas ( 0 a 3 ) de acordo com a presença ou intensidade do sintoma.

\section{Aplicados à criança}

Avaliação da pragmática da linguagem através do teste fonoaudiológico $A B F W$. O teste ABFW é um instrumento de que avalia quatro aspectos da linguagem infantil: fonologia, pragmática, vocabulário e fluência. Foi elaborado por 4 autoras, cujas iniciais foram o nome do teste (Andrade, Befi-Lopes, Fernandes Wertzner, 2000). No presente estudo, foi utilizado apenas o protocolo de avaliação da pragmática, elaborado por Fernandes (2000). O teste utilizado no estudo possui protocolo específico para avaliar atos comunicativos totais realizados pela mãe e pela criança; atos comunicativos somente da criança e atos comunicativos realizados por minuto (a quantidade de atos por minuto mostra, dentro dos parâmetros apontados pela autora, se a criança está com o seu 
desempenho dentro do esperado para a idade). Para amostras correspondentes da faixa etária estudada, os autores indicam como referência, aproximadamente, seis a oito atos por minuto. Também são avaliados os meios comunicativos utilizados: verbais (VE), aqueles que envolvem pelo menos $75 \%$ de fonemas da língua; e vocais (VO), referentes a todas as outras emissões e gestuais $(\mathrm{G})$, os quais envolvem movimentos de corpo e rosto. Nesta pesquisa, optamos por analisar os atos comunicativos totais realizados pela criança; atos comunicativos realizados por minuto (desempenho esperado para a idade), os meios comunicativos utilizados: verbais (VE), vocais (VO), e gestuais $(\mathrm{G})$.

\section{Procedimento}

A pesquisa foi aprovada no Comitê de Ética em Pesquisa da Secretaria Municipal de Saúde da Prefeitura do Município de São Paulo - Parecer no 0123/CEPS/ MS/2006 e do Comitê de Ética em Pesquisa do Hospital Universitário de São Paulo - Registro CEP-HU/ USP: 673/06 - SISNEP CAAE: 0051.0.198.000-06. Os participantes que fazem parte deste estudo aceitaram participar da pesquisa e assinaram o Termo de Consentimento Livre e Esclarecido.

\section{Coleta de dados}

As avaliações foram realizadas em uma sala de espelho duplo, desenvolvida no Instituto de Psicologia de uma Universidade em São Paulo para coleta de dados. Ela foi adaptada para o Projeto Ipê, e era previamente arrumada, com um tapete, quatro almofadas, uma caixa com brinquedos (com carinhos, jogo de chá de brinquedo, caixa de ferramentas, mini fazendinha, ônibus escolar de madeira, caminhão de madeira, animais da fazenda e da selva) e uma casinha de bonecas com duas famílias; duas câmeras digitais, uma em cada lado da sala.

\section{Protocolo de filmagem}

A mãe e sua criança de 36 meses eram convidadas a entrarem na sala de filmagens e a brincarem livremente na sala com os brinquedos disponíveis (descritos anteriormente), o experimentador retirava-se e as deixava sozinhas, sendo filmadas interagindo por 20 minutos. Findado esse tempo, o experimentador retornava à sala e solicitava que a mãe pedisse ao seu filho que a ajudasse a guardar os brinquedos na caixa, finalizando o momento de brincadeira e informando que iriam iniciar outra etapa. O protocolo para a tarefa de recolher os brinquedos foi baseado no trabalho desenvolvido por Kuczynski e Kochanska (1990) com algumas adaptações.
Um experimentador, formado em psicologia, foi treinado e ficou responsável por toda a coleta de dados e filmagens do Projeto Ipê no $3^{\circ}$ ano de vida da criança.

\section{Avaliação da interação mãe criança na tarefa de recolher os brinquedos}

Os vídeos foram analisados e os comportamentos tanto maternos quanto os de seus filhos, codificados. As observações dos comportamentos maternos foram classificadas em: diretos; indiretos e explicações. As observações dos comportamentos de resposta da criança à solicitação materna foram categorizadas por: 1) ocorrência da reação da criança quanto à obediência: obediência comprometida (adesão imediata à proposta da mãe), obediência situacional (adesão mediante insistências) e desobediência (não adesão); 2) por frequência dos diversos padrões emitidos pela criança nos casos de obediência situacional ou desobediência: ignorar o comando materno, recusas simples (dizer não), recusas rebuscadas (inventar desculpas) e desafio (teimar, discutir).

\section{Avaliação do aspecto pragmático da linguagem}

Todas as comunicações (todas as falas das mães e das crianças) filmadas nos 20 min da interação foram transcritas nos protocolos do teste de linguagem (ABFW). A transcrição e a análise das filmagens foram realizadas por uma das autoras deste trabalho, fonoaudióloga, especialista em linguagem infantil e treinada para utilizar o instrumento acima descrito. Foram analisadas as quantidades de atos comunicativos totais (a soma de atos realizados pela mãe e pela criança), a quantidade de atos comunicativos realizados somente pela criança. Com este, calculou-se a ocorrência de atos comunicativos por minuto (para verificação do desempenho da criança no teste). Verificaram-se também as ocorrências dos meios (vocal, verbal e gestual) durante o discurso da criança.

A codificação dos vídeos foi submetida à avaliação de dois juízes treinados na metodologia de análise, a fim de estabelecer o índice de fidedignidade das observações. Os juízes independentes receberam um manual contendo instruções de análise dos registros em vídeo, assim como o conjunto de definições de categorias de codificação. O índice de fidedignidade (Kappa) para avaliação do aspecto pragmático da linguagem foi de 0,88 e da tarefa de recolher os brinquedos foi de 0,87 .

Os resultados foram registrados no software SPSS (Statistical Package for Social Sciences) versão 20, utilizado para o processamento de análises. A 
análise dos dados foi realizada utilizando percentuais descritivos e testes estatísticos, como: testes quiquadrado de contingência, testes de média a fim de verificar a diferença entre mais de dois (MANOVA) grupos em função de uma ou mais variáveis contínuas dependentes e técnicas multivariadas (os dados acima descritos são variáveis consideradas correlacionadas). O nível de significância adotado foi de 5\%.

\section{Resultados}

\section{Avaliação dos aspectos pragmáticos da linguagem}

Comparando-se as médias dos grupos com e sem Depressão pós-parto (DPP), observou-que, embora não haja diferença significativa (Tabela 1), as díades do grupo com DPP falaram mais (atos comunicativos totais: com $\mathrm{DPP}=171,14$ e sem $\mathrm{DPP}=157,76)$; e as crianças do mesmo grupo interagiram mais com suas mães (com DPP $=85,89$ atos e sem DPP $=78,24)$. Ambos os grupos apresentaram desempenho esperado para a idade (atos comunicativos por minuto: com DPP 12 e sem DPP 12, veja Tabela 2).
O meio comunicativo utilizado por todas as crianças foi o verbal, com uma média de 75,96 verbalizações no grupo com DPP e 70,58 no grupo sem DPP. Os gestos e vocalizações foram realizados em menor quantidade por ambos os grupos. Em todos os meios, não houve diferença significativa entre os grupos.

Com relação às funções comunicativas, constatouse semelhança entre os grupos, ou seja, as crianças utilizaram funções comunicativas semelhantes, tais como: comentário, pedido de informação, pedido de ação, exibição, performativo e nomeação. A mais realizada foi comentário, seguido de nomeação e pedido de informação. Observou-se diferença significativa nas funções nomeação, em que o grupo sem DPP nomeou mais que o outro grupo (sem DPP 10.45 e com DPP $7.39 ; \mathrm{F}=4.16, p=.04)$ que, por sua vez, exibiu mais os objetos (com DPP 7.89 e sem DPP 5.39; $\mathrm{F}=5.00, p=.02$ ) para as suas mães que o grupo sem depressão (Tabela 1). Houve diferença significativa entre os grupos sem DPP $(\mathrm{M}=11.93, S D=12.06)$ e com DPP $(\mathrm{M}=11.10$, $S D=6.28)$ no pedido de informação [F=4.77, $p=.03]$. Embora as médias sejam muito parecidas, se percebe a diferença entre eles no desvio padrão.

TABELA 1

Comparação das mães com depressão e sem no desempenho de linguagem e das funções comunicativas das crianças no teste $\mathrm{ABFW}$ e respostas das díades na tarefa de recolher os brinquedos

\begin{tabular}{|c|c|c|c|c|}
\hline & Sem DPP & Com DPP & \multirow{2}{*}{$F$} & \multirow{2}{*}{$p$} \\
\hline & $M(S D)$ & $M(S D)$ & & \\
\hline \multicolumn{5}{|l|}{ Teste $A B F W$ - crianças } \\
\hline \multicolumn{5}{|l|}{ - Quantidade total de } \\
\hline Vocalizações & $3.30(3.80)$ & $3.32(4.03)$ & .00 & .97 \\
\hline Verbalizações & $70.58(32.70)$ & $75.96(29.06)$ & .30 & .58 \\
\hline Gestos & $5.76(7.55)$ & $7.86(8.12)$ & 1.01 & .31 \\
\hline Atos comunicativos & $78.24(34.51)$ & $85.89(31.40)$ & .63 & .42 \\
\hline Atos comunicativos totais & $157.76(61.95)$ & $171.14(56.98)$ & .80 & .37 \\
\hline Atos comunicativos por min & $4.99(2.22)$ & $5.62(1.95)$ & 1.03 & .31 \\
\hline Espaço comunicativo* & $48.69(5.31)$ & $46.62(11.49)$ & 3.79 & .05 \\
\hline Pedido de ação & $5.57(5.52)$ & $5.21(4.87)$ & 1.08 & .30 \\
\hline Pedido de informação* & $11.93(12.06)$ & $11.10(6.28)$ & 4.77 & .03 \\
\hline - Exibição* & $5.39(4.21)$ & $7.89(7.61)$ & 5.00 & .02 \\
\hline - Comentário & $27.96(16.58)$ & $33.82(14.58)$ & .03 & .85 \\
\hline - Nomeação* & $10.45(7.46)$ & $7.39(6.05)$ & 4.16 & .04 \\
\hline - Performativo & $5.15(6.32)$ & $5.64(6.78)$ & .00 & .98 \\
\hline \multicolumn{5}{|l|}{ Tarefa arrumar os brinquedos } \\
\hline \multicolumn{5}{|l|}{ - Comandos maternos } \\
\hline Comando direto* & $3.36(2.88)$ & $2.25(1.14)$ & 15.27 & .000 \\
\hline Comando indireto* & $1.06(1.56)$ & $.64(.78)$ & 4.25 & .04 \\
\hline Explicação* & $.82(1.23)$ & $.25(.44)$ & 13.73 & .000 \\
\hline \multicolumn{5}{|l|}{ - Respostas das crianças } \\
\hline Ignorar* & $3.61(10.81)$ & $1.04(5.28)$ & 5.46 & .02 \\
\hline Recusa simples* & $1.09(2.50)$ & $.18(.54)$ & 9.05 & .004 \\
\hline Recusa rebuscada* & $.12(.41)$ & - & 11.09 & .001 \\
\hline
\end{tabular}

DPP: depressão pós parto; M: Média; SD: desvio padrão. 
TABELA 2

Número de participantes e porcentagem das situações de obediência da criança na tarefa de recolher os brinquedos e atos comunicativos pelas crianças

\begin{tabular}{lccc}
\hline & $\begin{array}{c}\text { Sem DPP } \\
n(\%)\end{array}$ & $\begin{array}{c}\text { Com DPP } \\
n(\%)\end{array}$ & $p$ \\
Obediência comprometida & $12(36.4)$ & $22(78.6)$ & \\
Obediência situacional & $11(33.3)$ & $4(14.3)$ & \\
$\begin{array}{l}\text { Desobediência } \\
\begin{array}{l}\text { Atos comunicativos esperado } \\
\text { para a idade * }\end{array}\end{array}$ & $10(30.3)$ & $2(7.1)$ & .005 \\
\hline
\end{tabular}

* (6 a 8 atos comunicativos/min).

DPP: depressão pós parto.

Teste Qui-quadrado (entre as mães com e sem depressão).

\section{Avaliação do comportamento da díade na tarefa recolher os brinquedos}

\section{Observação do Comportamento Materno}

Diferenças significativas entre os grupos também podem ser notadas nos comandos maternos (Tabela 1). Observou-se que as mães sem depressão realizam significantemente mais comandos diretos (sem DPP $\mathrm{M}=3.36, S D=2.88$; com $\mathrm{DPP} \mathrm{M}=2.25, \mathrm{SD}=1.14$; $\mathrm{F}=15.27, p<.001$ ), indiretos (sem DPP $\mathrm{M}=1.06$, $S D=1.56 ;$ com $\mathrm{DPP} \mathrm{M}=.64, \mathrm{SD}=.78 ; \mathrm{F}=4.25, p<.05)$ e explicam mais as situações durante a interação do que as mães do outro grupo (sem DPP $\mathrm{M}=.82, S D=1.23$; com $\mathrm{DPP} \mathrm{M}=.25, S D=.44 ; \mathrm{F}=13.73, p<.001)$.

\section{Observação dos Comportamentos de resposta da criança à solicitação materna}

\section{A - Tipo de reação da criança quanto à obediência:}

Com relação à tarefa de recolher brinquedos, observou-se diferenças significativa no teste Quiquadrado $\left(\chi^{2}=10,56, p=.005\right)$ entre os grupos com e sem DPP. O grupo DPP $(78,6 \%)$ apresentou mais obediência comprometida que o outro grupo $(36,4 \%)$, enquanto que obediência situacional $(33,3 \%)$ e desobediência $(30,3 \%)$ foram realizadas em maior número pelo grupo sem depressão (Tabela 1).

\section{B - Frequência dos diversos padrões emitidos pela criança nos casos de obediência situacional ou desobediência}

Pode ser notada nas respostas das crianças (Tabela 1) que houve diferenças significativas entre os grupos (ignorar: $\mathrm{F}=5.46, p=.02$; fornecer recusas simples: $\mathrm{F}=9.05, p=.004$; fornecer recusas rebuscadas: $\mathrm{F}=11.09, p=.001$; e desafios: $\mathrm{F}=5.93$, $p=.01)$. As crianças de mães sem DPP recusam-se a fazer as tarefas, ignorando os comandos $(\mathrm{M}=3.61$, $S D=10.81)$, fornecem recusas simples $(\mathrm{M}=1.09$, $S D=2.50)$, fornecem recusas rebuscadas $(\mathrm{M}=.12$, $S D=.41)$ e desafiam suas mães $(\mathrm{M}=.12, S D=.54)$ do que as crianças cujas mães não apresentam DPP (Tabela 1). Esses dados podem ser melhor observados na Tabela 2 em que as crianças do grupo com DPP apresentaram mais obediência comprometida (78.6\%) e situacional (14.3\%), e conforme mostra a Tabela 1, mesmo com mais comandos e explicações, as crianças do grupo sem DPP recusam-se mais a fazer as tarefas propostas (desobediência $=30.3 \%$ ).

Foi realizado o teste MANOVA para observar o efeito dos comportamentos maternos e da depressão nas respostas das crianças de ambos os grupos. Constatou-se que os filhos, cujas mães oferecem mais explicações, exibem menos os objetos que estão brincando $(\mathrm{F}=5.00 ; p=.02)$, recusam-se mais a realizar as atividades $(\mathrm{F}=9.05 ; p=.004)$ e oferecem mais desculpas às mães $(\mathrm{F}=11.09 ; p=.001)$. Há o efeito do comando indireto das mães nos comentários realizados pelas crianças durante a interação $(\mathrm{F}=4.18 ; p=.04)$, na recusa em realizar as atividades $(\mathrm{F}=14.98 ; p=.000) \mathrm{e}$ as crianças desafiam mais as mães durante a atividade realizada por ambos $(\mathrm{F}=4.89 ; p=.03)$. As crianças que se recusaram a realizar as atividades foram aquelas também cujas mães realizaram mais comandos diretos $(\mathrm{F}=9.41 ; p=.003)$.

Observa-se, com os dados acima descritos que as mães do grupo sem depressão fornecem mais comandos diretos e, se não obtém resultados, elas explicam para as crianças o que deve ser feito. As crianças, por sua vez, durante a interação, parecem realizar trocas comunicativas com suas mães através de comentários, nomeações e recusas rebuscadas.

\section{Discussão}

Através de um recorte do estudo longitudinal, observou-se, neste estudo o desempenho social e de linguagem de crianças de 36 meses de idade cronológica. Não foi encontrado efeito significativo da DPP na avaliação da linguagem utilizando o teste ABFW nas crianças do estudo.

Em linhas gerais, possíveis alterações ou falhas na comunicação podem aparecer em idade mais avançada, como mostram os estudos de Murray et al. (2010), desta forma, agregou-se ao estudo a interação da díade na tarefa de recolher os brinquedos, para observar o efeito do comportamento materno na funcionalidade da linguagem infantil e a influencia da DPP neste processo. As crianças do grupo DPP foram ligeiramente mais 
comunicativas em geral. Algumas hipóteses foram levantadas para este desempenho. Motta et al. (2005), Silva et al. (2010) e Schmid et al. (2011) relatam que os sintomas depressivos comprometem a disponibilidade cognitiva e emocional da mãe, determinando que a mãe não seja adequadamente responsiva à criança, pela falta de contingência, de engajamento e de trocas ajustadas. As crianças, para chamarem a atenção da mãe, comunicam-se de todas as formas possíveis (gestos, vocalizações e verbalizações) e, conforme observado nos resultados, exibem mais os objeto da brincadeira que o outro grupo que, por sua vez, nomeiam mais os utensílios que estão utilizando. Radke-Yarrow (1998) relata que as crianças expressam um modo particular de enfrentar as condições da mãe, sendo uma boa criança. Isso pode ser observado no comportamento prossocial em que as crianças do grupo depressivo se mostraram mais obedientes, enquanto que as do outro grupo mostraram obediência situacional e desobediência, além de recusa aos comandos maternos.

Fonseca, Silva e Otta (2010) acrescentam que as crianças, em condições adversas, apresentam mecanismos compensatórios. De Felipe, Bussab e Vieira (2016) observaram, em seu estudo que as mães com sintomas depressivos apresentam-se significantemente diferentes das mães não-depressivas com relação à práticas e crenças parentais. As mães do primeiro grupo obtiveram escores menores de interdependência e autonomia, seguindo o modelo de etnoteoria de cuidados parentais proposto por Keller (2003), o que poderia explicar a menor quantidade de comandos verbais maternos e a maior média de obediência das crianças. As crianças do grupo sem depressão, dessa forma podem ser mais autônomas, exigindo mais atenção da mãe. Outra hipótese levantada seria que, por haver sintomas depressivos associados à interação da mãe com a criança, as crianças podem ter recebido estímulos ambientais (outros cuidadores, creche) em maior quantidade que as crianças do grupo sem DPP.

Os achados deste estudo merecem um entendimento mais pormenorizado e contextualizado em futuras investigações com diferentes faixas etárias e avaliações realizadas por outros pesquisadores do projeto. Uma limitação do estudo foi a diminuição da amostra por falta de algum dado durante o processo da pesquisa longitudinal ou avaliação tanto da mãe quanto das crianças.

\section{Conclusão}

Com base nos achados do presente estudo, reforça-se a importância de mais estudos na área para verificação mais pormenorizada dos efeitos da DPP na linguagem e nos comportamentos maternos e infantis. Esses trabalhos são fundamentais para organização de programas de orientação às gestantes, puérperas e intervenções precoces. Observou-se que não houve a influência da depressão materna no desenvolvimento da linguagem das crianças de três anos, contrariando os dados encontrados na literatura. Pelos vários indicadores, a presente pesquisa reitera a importância da mãe no desenvolvimento da criança - sem diminuir a importância das demais pessoas do contexto familiar e nem da rede social materna e da criança. Entende-se que mães com DDP podem ser agentes do desenvolvimento, pois, mesmo com as limitações impostas pela própria DDP, se mostraram, neste estudo, provedoras para seus filhos.

\section{Referências}

Andrade, C. R. F, Befi-Lopes, D. M., Fernandes, F. D. M. \& Wertner, H. F. (2000). ABFW-Teste de linguagem infantil nas áreas de fonologia, vocabulário, fluência e pragmática. Barueri: Pró-Fono.

American Psychiatric Association. (2014). DSM-V. Manual de Diagnóstico e Estatística dos Distúrbios Mentais (5aㅡ. ed.). Porto Alegre: Artmed.

Brocchi, B. S. \& Bussab, V. S. R. (2015). Interação mãe-criança e desenvolvimento da linguagem: a influência da Depressão pós-parto. Psico (Porto Alegre), 43(1), 103-111.

Brum, E. H. M. \& Schermann, L. (2006). O impacto da depressão maternal nas interações iniciais. Psico (Porto Alegre), 37(2), 151-158.

Campbell, S. B., Matestic, P., von Stauffenberg, C., Mohan, R., \& Kirchner, T. (2007). Trajectories of maternal depressive symptoms, maternal sensitivity, and children's functioning at school entry. Developmental Psychology, 43(5), 1202-1215. https://doi.org/10.1037/0012-1649.43.5.1202

Cox, J. L., Holden, J. M., \& Sagovsky, R. (1987). Detection of Postnatal Depression: Development of the 10 Item Edinburg Postnatal Depression Scale. British Journal of Psychiatry, 150, 782-786. https://doi.org/10.1192/bjp.150.6.782

Cruz, E. B. S., Simões, G. L., \& Faisal-Cury, A. (2005). Rastreamento da depressão pós-parto em mulheres atendidas pelo Programa de Saúde da Família. Revista Brasileira de Ginecologia e Obstetrícia, 27, 181-188. https://doi.org/10.1590/ S0100-72032005000400004 
Da Silva, V. A., Moraes-Santos, A. R., Carvalho, M. S., Martins, M. L., \& Teixeira, N. A. (1998). Prenatal and postnatal depression among low income Brazilian women. Brazilian Journal of Medical and Biological Research, 31, 799-804. https://doi.org/10.1590/S0100-879X1998000600012

De Felipe, Bussab, V. S. R., \& Vieira, M. L. (2016). Relationship between postpartum depression and maternal perceptions about ethnotheories and child rearing practices. Early Child Development and Care, 186(6).

Field, T., Diego, M., Hernandez-Reif, M., Figueiredo, B., Ezell, S., \& Siblalingappa, V. (2010). Depressed mothers and infants are more relaxed during breastfeeding versus bottlefeeding interactions: brief report. Infant Behavior and Development, 33, 241-244. https://doi.org/10.1016/j.infbeh.2009.12.006

Fonseca, V. R. J. R. M., Silva, G. A., \& Otta, E. (2010). Relação entre depressão pós-parto e disponibilidade emocional materna. Cadernos de Saúde Pública, 26(4),738-746. https://doi.org/10.1590/S0102-311X2010000400016

Goodman, S. H., Brogan, D., Lynch, M. E. \& Fielding, B. (1993). Social and emotional competence in children of depressed mothers. Child Development, 64, 516-531. https://doi.org/10.2307/1131266

Goodman, S. H. \& Garber, J. (2017). Evidence-based interventions for depressed mothers and their young children. Child Development, 88(2), 368-377. https://doi.org/10.1111/cdev.12732

Hammond, S. I. \& Carpendale, J. I. M. (2015). Helping children help: The relation between maternal scaffolding and children's early help: Helping children help. Social Development, 24, 367-383. https://doi.org/10.1111/sode. 12104

Hatzinikolaou, K. \& Murray, L. (2010). Infant sensitivity to negative maternal emotional shifts: effects of infant sex, maternal postnatal depression, and interactive style. Infant Mental Health Journal, 31(5), 591-610. https://doi. org/10.1002/imhj.20265

Klaus, M. H. \& Klaus, P. H. (2001). Seu Surpreendente recém-nascido. Porto Alegre. Artmed.

Keller, H., Papaligoura, Z., Kuensemueller, P., Voelker, S., Papaeliou C., Lohaus, A., Lamm, B., Kokkiaki, T., Chrysikou, E., \& Mousouli, V. (2003). Concepts of mother-infant interaction in Greece and Germany. Journal of Cross-Cultural Psychology, 34(6), 677-689. https://doi.org/10.1177/0022022103257035

Kuczynski, L. \& Kochanska, G. (1990). Development of children's noncompliance strategies from toddlerhood to age 5. Developmental Psychology, 26(3), 398-408. https://doi.org/10.1037/0012-1649.26.3.398

Lopes, E. R., Jansen, K., Quevedo, L. A., Vanila, R. G., Silva, R. A., \& Pinheiro, R. T. (2010). Depressão pós-parto e alterações de sono aos 12 meses em bebês nascidos na zona urbana da cidade de Pelotas/RS. Jornal Brasileiro de Psiquiatria, 59(21), 88-93. https://doi.org/10.1590/S0047-20852010000200002

Lovejoy, M. C., Graczyk, P. A., O’Hare, E., \& Neuman, G. (2000). Maternal depression and parenting behavior: a meta-analytic review. Clinical Psychology Review, 20(5), 561-592. https://doi.org/10.1016/S0272-7358(98)00100-7

Morais, M. L. S., Lucci, T., \& Otta, E. (2013). Postpartum depression and child development in first year of life. Estudos em Psicologia, 30(1), 7-17. https://doi.org/10.1590/S0103-166X2013000100002

Motta, M. G., Lucion, A. B., \& Manfro, G. G. (2005). Efeitos da Depressão materna no desenvolvimento neurobiológico e psicológico da criança. Revista de Psiquiatria do Rio Grande do Sul, 27(2). https://doi.org/10.1590/S010181082005000200007

Murray, L., Arteche, A., Fearon, P, Halligan, S., Croudace, T., \& Cooper, P. (2010). The effects of maternal postnatal depression and child sex on academic performance at age 16 years: a developmental approach. Journal of child Psychology and Psychiatry, 51(10), 1150-1159. https://doi.org/10.1111/j.1469-7610.2010.02259.x

Pettygrove, D. M., Hammond, S. I., Karahuta, E. L., Waugh, W. E., \& Brownell, C. A. (2013). From cleaning up to helping out: Parental socialization and children's early prosocial behavior. Infant Behavior \& Development, 36 , 843-846. https://doi.org/10.1016/j.infbeh.2013.09.005

Putallaz, M. (1987). Maternal behavior and children's sociometric status. Child Development, 58(2), 324-340. https:// doi.org/10.2307/1130510

Quevedo, L. A., Silva, R. A., Godoy, R., Jansen, K., Matos, M. B., Pinheiro, K. A. T., \& Pinheiro, R. T. (2011) The impact of maternal post-partum depression on the language development of children at 12 months. Child: care, health and development, 38(3), 420-424. https://doi.org/10.1111/j.1365-2214.2011.01251.x

Radke-Yarrow, M. (1998). Children of depressed mothers: from early childhood to maturity. New York: Cambridge University Press.

Radke-Yarrow, M., Cummings, E. M., Kuczynski, L. \& Chapman, M. (1985). Patterns of attachment in two and threeyear-olds in normal families and families with parental depression. Child Development, 56, 884-893. https://doi. org/10.2307/1130100

Santos, M. F. S., Martins, F. C. \& Pasquali, L. (1999). Escalas de auto-avaliação de depressão pós-parto: estudo no Brasil. Revista de Psiquiatria Clínica, 26(2), 32-40.

Schmid, B., Blomeyer, D., Buchmann, A.F., Trautmann-Villalba, P., Zimmermann, U.S., Schmidt, M.H., Esser, G, Banaschewski, T \& Laucht, M. (2011): Quality of early mother-child interaction associated with depressive psychopathology in the offspring: A prospective study from infancy to adulthood. Journal of Psychiatric Research, 45, 1387-1394. https://doi.org/10.1016/j.jpsychires.2011.05.010

Schmidt, E. B., Piccoloto, N. M. \& Müller, M. C. (2005). Depressão pós-parto: fatores de risco e repercussões no desenvolvimento infantil. Psico-USF, 10(1), 61-68. https://doi.org/10.1590/S1413-82712005000100008 
Schwengber, D. D. S \& Piccinini, C. A. (2003): O impacto da depressão pós-parto para interação mãe-bebê. Estudos de Psicologia, 8(3),403-411. https://doi.org/10.1590/S1413-294X2003000300007

Schwengber, D. D. S. \& Piccinini, C. A. (2005). A experiência da maternidade no contexto da depressão materna no final do primeiro ano de vida do bebê. Estudos de Psicologia, 22(2), 143-156. https://doi.org/10.1590/S0103166X2005000200004

Seidl-de-Moura, M. L. \& Ribas, A. F. P. (2004). Evidência sobre características de bebês recém-nascidos: um convite a reflexões teóricas. In M. L. Seidl-de-Moura. O bebê do século XXI e a psicologia em desenvolvimento (pp. 21-60). São Paulo: Casa do Psicólogo.

Silva, F. C. S., Araújo, T. M., Araújo, M. F. M., Carvalho, C. M. L., \& Caetano, J. A. (2010): Depressão pós-parto em puérperas: conhecendo interações entre mãe, filho e família. Acta Paulista de Enfermagem, 23(3), 411-416. https:// doi.org/10.1590/S0103-21002010000300016

Silva, G. A. (2013). Prevalência de depressão pós-parto em países desenvolvidos e em desenvolvimento: contribuições metodológicas de uma metanálise. Tese [Doutorado], Instituto de Psicologia, Universidade de São Paulo, São Paulo.

Skafuzka E. (2000). Prevalência da depressão pós-parto e fatores associados em mães que deram à luz em um Hospital Universitário do Município de S. Paulo. Tese [Doutorado], Faculdade de Medicina, Universidade de São Paulo, São Paulo.

Tronick, E. Z. \& Gianino, A. F. (1986). The transmission of maternal disturbance to the infant. New Directions for Child and Adolescent Development, 34, 5-11. https://doi.org/10.1002/cd.23219863403

Wachs, T. D., Black, M. M. \& Engle, P. L. (2009). Maternal depression: a global threat to children's health, development, and behavior and to human rights. Child Development Perspectives, 3(1), 51-59. https://doi.org/10.1111/j.17508606.2008.00077.x

Zaconeta, A. M., Motta, L. D. \& França, P. S. (2004) Depresión postparto: prevalencia de test de rastreo positivo en puérperas del Hospital Universitário de Brasília, Brasil. Child Obstet Ginecol, 69, 209-213.

\footnotetext{
Dados dos autores:

Vera Silvia Raad Bussab - Doutora, Universidade de São Paulo.

Endereço para correspondência:

Laura Cristina Stobäus

Pontifícia Universidade Católica do Rio de Janeiro

Departamento de Educação

Rua Marques de São Vicente, 225 sala 1052

22451-900 Rio de Janeiro, RJ, Brasil

<laustoba@gmail.com>
}

Laura Cristina Stobäus - Doutora, Pontifícia Universidade Católica do Rio de Janeiro.

Beatriz Servilha Brocchi - Doutora, Pontifícia Universidade Católica de Campinas.

Recebido em: 24.08 .2017

Aceito em: 07.05 .2018 